\title{
Møller's energy of the Kerr-NUT Metric
}

\author{
Gamal G.L. Nashed \\ Mathematics Department, Faculty of Science, Ain Shams University, Cairo, Egypt \\ e-mail:nashed@asunet.shams.edu.eg
}

'The energy distribution of the Kerr-NUT space-time is calculated using Møller's energymomentum complex within the framework of the Riemannian geometry.

PACS: 04.20.Cv, 04.20.Fy

Keywords: Kerr-NUT space-time, Møller's energy-momentum complex.

\section{Introduction}

The search for a consistent expression for the gravitating energy and angular momentum ' of a self-gravitating distribution of matter is undoubtedly a long-standing problem in general relativity (GR). It is believed that the energy of the gravitational field is not localizable, i.e., 'defined in a finite region of the space. The gravitational field does not possess the proper definition of an energy momentum tensor and one usually defines some energy-momentum as 'Tolamn [1], Landau-Lifschitz [2], Goldberg [3], Bergmann [4], Møller [5], Komar [6], Arnowit et al. [7] Nahmed-Achar and Schutz [8], Bratnik [9], Kataz and Ori [10] etc. which are pseudo-tensors and depend on the second derivative of the metric tensor. These quantities can be annulled by an adequate transformation of coordinate. They $[2,4]$ justify the results as being consistent with Einstein's principle of equivalence. In this principle, it can be always find a small region of space-time, where it prevails Minkowski space-time. In such a spacetime, energy of the gravitational field is null. Therefore, it is only possible to define the energy of the gravitational filed in whole space-time region and not only in a small region.

Møller [5, 11] proposed an expression which is the best to make calculations in any coordinate system within the framework of Riemannian geometry. He claimed that his expression would give the same results for the total energy and momentum as the Einstein's energymomentum complex for a closed system. Lessner [12] gave his opinion that Møller's definition is a powerful concept of energy and momentum in (GR). However, Møller prescription was also criticized by some researchers [13] [16]. Komar's complex [16], though not restricted to the use of Cartesian coordinate, is not applicable to non-static space-times. Thus each 
of these energy-momentum complex has its own drawbacks. As a result, these ideas of the energy-momentum complexes could not lead to some unique definition of energy in (GR).

Several attempts have been made to resolve the problem of energy-momentum localization but still remains out of hand. This problem first appeared in electromagnetic which turns out to be a serious matter in GR due to the non-tensorial quantities. Virbhadra et al. [17] explored several space-times for which different energy-momentum complexes show a high degree of consistency in giving the same and acceptable energy-momentum distribution. Aguirregabira et al. [18] showed that five different energy-momentum complexes gave the same result for any Kerr-Schild class (including the Schwarzschild, Reissner-Nordström, Kerr and Vaidya metrics). An extension of these calculations has been done [19] [20].

It is the aim of the present study to calculate the energy, momentum and angular momentum of the Kerr-NUT space-time using the energy-momentum complex given by Møller [5] within the framework of GR. In $§ 2$, we give a brief review of Kerr-NUT space-time. Calculation of energy using the energy-momentum complex of Møller is given in §3. A special cases of the energy is also discussed in $\S 3$. Final section is devoted to main results.

\section{Kerr-NUT space-time}

The Kerr-NUT (Newman-Unti-Tamburino) space-time [21] describes a stationary axi- symmetric object with gravitomagnetic monopole and dipole moments associated with nonzero values of the NUT and Kerr parameters $L$ and $a$ respectively and as such a useful model for exploring gravitomagnetism [22]. The Kerr-NUT space-time and its spacial cases all belong to the larger class of stationary axi-symmetric type D vacuum solutions of the Einstein equations found by Carter [23] for which the Hamilton-Jacobi equation for the geodesic is separable. The stability of the Kerr-NUT space-time is probed by studying their perturbation by fields of various spin [24, 25]. The Kerr-NUT metric in Boyer-Lindquist-like coordinates is given by the following line-element

$$
\begin{aligned}
d s^{2}= & \frac{1}{\Sigma}\left\{\Delta-a^{2} \sin ^{2} \theta\right\} d t^{2}-\frac{\Sigma}{\Delta} d \rho^{2}-\Sigma d \theta^{2}-\frac{1}{\Sigma}\left\{(\Sigma+a \chi)^{2} \sin ^{2} \theta-\chi^{2} \Delta\right\} d \phi^{2} \\
& -\frac{2}{\Sigma}\left\{\chi \Delta-a(\Sigma+a \chi) \sin ^{2} \theta\right\} d t d \phi,
\end{aligned}
$$

and the corresponding electromagnetic Faraday tensor can be expressed in terms of the 2 -form

$$
\begin{aligned}
\mathbf{F}= & \frac{Q}{\Sigma^{2}}\left[\left(\rho^{2}-\{l+a \cos \theta\}^{2}\right) d \rho \wedge(d t-\chi d \phi)\right. \\
& \left.+2 \rho \sin \theta(l+a \cos \theta) d \theta \wedge\left(\left\{\rho^{2}+a^{2}+l^{2}\right\} d \phi-a d t\right)\right],
\end{aligned}
$$

where $\Sigma, \Delta$, and $\chi$ are defined by

$$
\Sigma=\rho^{2}+(l+a \cos \theta)^{2}, \quad \Delta=\rho^{2}-2 M \rho-l^{2}+a^{2}+Q^{2}, \quad \chi=a \sin ^{2} \theta-2 l \cos \theta .
$$


Units are chosen such that $G=c=1$, so that $(M, Q, a, l)$ all have the dimension of length: the source has mass $\mathrm{M}$, electric charge $\mathrm{Q}$, angular momentum $J=M a$ (i.e., gravitomagnetic dipole moment) along the z-direction, and gravitomagnetic monopole moment $\mu=-l$, where $l$ is the NUT parameter.

\section{Møller energy-momentum complex}

Møller's energy-momentum complex is given by

$$
\jmath_{\mu}{ }^{\nu}=\frac{1}{\kappa} \chi_{\mu, \rho}^{\nu \rho}
$$

satisfying the local conservation laws

$$
\frac{\partial \jmath_{\mu}{ }^{\nu}}{\partial x^{\nu}}=0
$$

where the antisymmetric superpotential $\chi_{\mu}{ }^{\nu \rho}$ is defined by

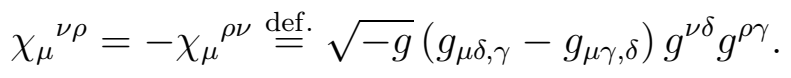

The energy and momentum components are given by

$$
P_{\mu}=\iiint \jmath_{\mu}{ }^{0} d x^{1} d x^{2} d x^{3}
$$

where $P_{0}$ is the energy while $P_{\alpha}$ stand for the momentum components. Using the Gauss's theorem the energy $E$ for a stationary metric is given by

$$
E=\frac{1}{8 \pi} \iint \chi_{0}{ }^{0 \alpha} n_{\alpha} d S
$$

where $n_{\alpha}$ is the outward unit normal vector over an infinitesimal surface element $d S$.

As is clear from Eq. (8) that the only required component is $\chi_{0}{ }^{01}$. Using Eq. (6) in Eq. (1) we get

$$
\chi_{0}{ }^{01}=\frac{2 \sin \theta\left(\rho^{2}+a^{2}+l^{2}\right)}{\left\{\rho^{2}+(a \cos \theta+l)^{2}\right\}^{2}}\left[M \rho^{2}-\rho Q^{2}-M a^{2} \cos ^{2} \theta-M l^{2}+2 \rho l^{2}+2 \rho a l \cos \theta\right],
$$

using Eq. (9) in Eq. (8) we get

$$
E_{M \varnothing l}=M+\frac{2 l^{2}}{\rho}-\frac{Q^{2}}{2 \rho}\left[1+\left\{\frac{\left(a^{2}+l^{2}+\rho^{2}\right)}{2 a \rho}-\frac{a l^{2}}{\rho Q^{2}}\right\}\left(\tan ^{-1}\left\{\frac{a-l}{\rho}\right\}+\tan ^{-1}\left\{\frac{a+l}{\rho}\right\}\right)\right] .
$$


The formula of energy given by Eq. (10) is a general formula and reduces to:

i) The Kerr-Newman energy when the NUT parameter $l=0[19,26]$.

ii) The Kerr energy when $Q=l=0[26]$.

iii) The Reissner-Nordström energy when $a=l=0$ [27].

iv) The Schwarzschild energy when $a=l=Q=0$ [28].

\section{Main results and discussion}

In GR, several attempts to obtain a meaningful and generally covariant expression for the energy density have proven unsuccessful [29]. The canonical energy-momentum pseudotensor expression derived from variational formulation of general relativity has been found neither unique nor tensorial. Although Komar [6] and Penrose [30] have developed some sophisticated approach, the canonical energy-momentum pseudotensors have been found useful in the computation of integrated energy, provided some restriction is imposed. Komar [6] definition is not restricted to "Cartesian coordinate" like one has for the Tolamn [1], LandauLifschitz [2], Goldberg [3], Bergmann [4]. However, Komar definition is applicable only to stationary space-times. The Møller energy-momentum complex is neither restricted to the use of particular coordinates nor to the stationary space-times. Lessner [12] pointed out that Møller definition is a powerful representation of energy and momentum in GR.

For our background metric given by Eq. (1) we have examined Møller pseudotensor. Then the total energy associated with metric given by Eq. (1) contained in a sphere of radius $\rho$ can be found as given by Eq. (10). This calculation shows that the total energy associated with the space-time given by Eq. (1) is shared by its exterior as well as interior. In the case of Kerr black hole, i.e., case (ii) above, it is clear from Eq. (10) that there is no energy contained by the exterior of the Kerr black hole and hence the entire energy

is confined with its interior. This result is quite in conformity with that obtained before $[17,26,29]$. 


\section{References}

[1] R.C. Tolman, Phys. Rev. 35 (1930), 875.

[2] L.D. Landau and E.M. Lifshitz, The Classical Theory of Fields (Pergamon Press, Oxford, 1980).

[3] J.N. Goldberg, Phys. Rev. 111 (1958), 315.

[4] P.G. Bergmann and R. Thomson, Phys. Rev. 89 (1953), 401; P.G. Bergman, Phys. Rev. 112 (1958), 287.

[5] C. Møller, Ann. Phys. (N.J.) 4 (1958), 347.

[6] A. Komar, Phys. Rev. 113 (1959), 934.

[7] R. Arnowitt, S. Deser and C.W. Misner, Phys. Rev., 122 (1961) 997.

[8] E. Nahmad-Achar and B.F. Schutz, Gen. Relat. Grav. 19 (1987), 7.

[9] R. Bartink, Phys. Rev. Lett. 62 (1989), 2346.

[10] J. Katz and A. Ori, Class. Quant. Grav. 7 (1990), 787.

[11] C. Møller, Ann. Phys. (N.J.) 12 (1961), 118.

[12] G. Lessner, Gen. Relat. Grav. 28 (1996), 527.

[13] H. Bondi, Proc. R. Soc. Lond. A427 (1990), 249.

[14] D. Kovacs, it Gen Relat. Grav. 17 (1985), 927.

[15] J. Novotny, Gen. Relat. Grav. 19 (1987), 1043.

[16] A. Komar, Phys. Rev. 113 (1959), 934.

[17] N. Rosen and K.S. Virbhadra, Gen. Relat. Grav. 26 (1993), 429; K.S. Virbhadra, Phys. Rev. D41 (1990), 1086; Phys. Rev. D42 (1990), 1066; Phys. Rev. D60 (1999), 104041; Phys. Rev. D42 (1990), 2919; K.S. Virbhadra and J.C. Parikh, Phys. Lett. B317 (1993), 312; Phys. Lett. B331 (1994), 302.

[18] J.M. Aguirregabiria, A. Chamorro and K.S. Virbhadra and J.C. Parikh, Gen. Relat. Grav. 28 (1996), 1393.

[19] S.S. Xulu, Int. J. Mod. Phys. A15 (2000), 1979; Mod. Phys. Lett. A15 (2000), 1151.

[20] I. Radinschi, Mod. Phys. Lett. A15 (2000), 803.

[21] E.T. Newman, L. Tamburino and T.Unti J. Math. Phys. 4 (1963), 915.

[22] D. Bini, C. Cherubini, R.T. Jantzen and B. Mashhoon, Phys. Rev. D67 (2003), 084013.

[23] B. Carter, Commun. Math. Phys. 10 (1968), 280. 
[24] D. Bini, C. Cherubini, R.T. Jantzen and R. Ruffini, Prog. Theor. Phys. 107 (2002), 1.

[25] D. Bini, C. Cherubini and R.T. Jantzen Class. Quant. Grav. 19 (2002), 1.

[26] G.G.L Nashed, Mod. Phys. Lett.A22(2007), 1047; Chaos, Soliton and Fractal 15 (2003), 687.

[27] G.G.L. Nashed and T. Shirafuji, Int. J. Mod. Phys. D16 (2007), 65; G.G.L. Nashed, Mod. Phys. Lett. A21 (2006), 2241.

[28] T. Shirafuji, G.G.L. Nashed, and K. Hayashi, Prog. Theor. Phys. 95 (1996), 665.

[29] M. Ahmed and S. M. Hossain , Prog. Theor. Phys. 93 (1995), 901.

[30] R. Penrose, Proc. R. Soc. London A381 (1982), 53. 